\title{
Morphological dependence in the spatial orientations of Local Supercluster galaxies
}

\author{
B. Aryal and W. Saurer
}

\author{
Institut für Astrophysik, Universität Innsbruck, Technikerstraße 25, 6020 Innsbruck, Austria \\ e-mail: [binil.aryal; walter.saurer]@uibk.ac.at
}

Received 16 July 2004 / Accepted 8 November 2004

\begin{abstract}
We have compiled a database of 4073 morphologically identified galaxies with radial velocity $<3000 \mathrm{~km} \mathrm{~s}^{-1}$ and examined the alignments of the spin vectors of these galaxies in the Local Supercluster (LSC). Our aim is to test the morphological dependence of galaxy orientation. The "position angle - inclination" method is used to find the polar and azimuthal angles of the galaxy rotation axes. The spatial isotropic distribution is assumed to examine the non-random effects. We have carried out chi-square, Fourier, and auto correlation tests to examine non-random effects in the distributions of the polar and azimuthal angles of the galaxy rotation axes. We suspect that the orientations of galaxies in the LSC could be closely related to galactic morphology. The spiral galaxies exhibit anisotropy although not at a very high significance level. It is found that the spin vector orientations of the spiral galaxies in the LSC tend to lie perpendicular to the LSC plane. No preferred orientation is found in the spin vector orientations of barred spiral and irregular galaxies. The orientations of early-type and late-type spirals and barred spirals are discussed.
\end{abstract}

Key words. galaxies: evolution - galaxies: formation - galaxies: statistics - galaxies: clusters: general astronomical data bases: miscellaneous

\section{Introduction}

The spin vector (SV hereafter) orientation of galaxies in the Local Supercluster (LSC hereafter) has been a subject of many investigations (e.g. Reinhardt \& Roberts 1972; Jaaniste \& Saar 1978, hereafter JS; MacGillivray et al. 1982; Kapranidis \& Sullivan 1983; MacGillivray \& Dodd 1985a,b; Dekal 1985; Flin \& Godlowski 1986 (FG); Kashikawa \& Okamura 1992 (KO); Godlowski 1993 (Go93); Godlowski 1994 (Go94); Hu et al. 1995; Yuan et al. 1997; Hu et al. 1998; Godlowski \& Ostrowski 1998). Different authors have drawn different conclusions. A few authors concluded that the SVs of galaxies tend to lie in the LSC plane (e.g. JS; FG; Go93; Go94), others showed that the SVs of galaxies tend to orient perpendicular to the LSC plane (e.g. Reinhardt \& Roberts 1972; MacGillivray et al. 1982; Hu et al. 1995). KO noticed a bimodal tendency, that is, galaxies near the LSC plane tend to have their SVs parallel to the LSC plane, while those off the LSC plane have their SVs perpendicular to it. MacGillivray et al. (1982) found a marginally significant tendency for galaxies to be parallel to the LSC plane with some secondary dependence on the supergalactic latitude. Kaprandis \& Sullivan (1983) noticed no significant effects. These inconsistencies are probably due to different methods and selection criteria applied by the authors.

Studies of the morphological dependence of galaxy orientation have been carried out by several authors.
MacGillivray \& Dodd (1985a) observed systematic effects both in alignment and winding direction of LSC spirals, the effects being strongest for the intermediate types. Dekal (1985) conducted a thorough study of possible alignments of the UGC (Nilson 1973) and ESO (Lauberts 1982) galaxies. In some subsamples (ellipticals) he detected alignments. However the results were mostly negative. Helou (1984) carried out an interesting study of the mutual orientations of the SVs in spiral pairs. He found that the SVs tend to be antiparallel and that the effect was stronger for pairs with a lower mass-to-luminosity ratio.

KO found that the SVs of early-type spiral LSC galaxies tend to lie parallel to the LSC plane whereas the late-type spirals show isotropy. A morphological dependence of the orientations of disk galaxies has been found for the LSC bright field galaxies (Hu et al. 1995). They noticed that the SVs of S and S0 galaxies tend to lie perpendicular to the LSC plane. Yuan et al. (1997) noticed that the SVs of spirals in the field galaxies tend to lie in the LSC plane. No preferred orientation was noticed for bright isolated field spiral galaxies (Hu et al. 1998).

In the Coma cluster Wu et al. (1997) found that the SVs of spiral galaxies tend to lie parallel or perpendicular to the cluster plane. Anisotropy was found by Flin (2001) when analysing 622 spiral galaxies in the Coma cluster. He noticed that the $\mathrm{SV}$ orientations of spiral galaxies in the Coma cluster tend to lie in the cluster plane. 
Table 1. The list of catalogues used.

\begin{tabular}{lrrl}
\hline \hline Catalogue & $N_{1}$ & $N_{2}$ & Ref. \\
\hline NGC & 1834 & 302 & Dreyer (1895-1908) \\
UGC & 1098 & 194 & Nilson (1973) \\
ESO & 546 & 114 & Lauberts (1982) \\
IC & 421 & 129 & Dreyer (1895-1908) \\
MCG & 187 & 183 & Vorontsov-Vel'Yaminov et al. (1962-74) \\
UGCA & 167 & 81 & Nilson (1974) \\
VCC & 100 & 100 & Binggeli et al. (1985) \\
CGCG & 89 & 85 & Zwicky et al. (1961-68) \\
KUG & 58 & 56 & Takase (1980-2000) \\
MRK & 40 & 40 & Markarian (1967) \\
MESSIER & 41 & 8 & Messier (1784) \\
BCG & 28 & 28 & Brandner et al. (2000) \\
LSBG & 23 & 23 & Impey et al. (1996) \\
SBS & 19 & 18 & Markarian et al. (1983) \\
LCRS & 18 & 18 & Shectman et al. (1996) \\
DDO & 16 & 13 & Bergh (1959-1966) \\
IRAS & 15 & 15 & Infrared Astronomical Satellite 1983 \\
SGC & 11 & 9 & Corwin et al. (1985) \\
UM & 11 & 10 & MacAlpine et al. (1977a-1981) \\
CCC & 10 & 10 & Doi et al. (1995) \\
\hline
\end{tabular}

$N_{1}$ : total number of LSC galaxies.

$N_{2}$ : number of galaxies the PAs of which were measured on the ESO and POSSII films by the authors.

In this paper we analyse the spatial orientations of 4073 morphologically identified galaxies in the LSC. We test the following: (1) Do the orientations of spiral, barred spiral and irregular galaxies in the LSC show a difference; (2) Is there any correlation between the spatial orientations of early-type and late-type spirals and barred spirals and finally; (3) Does a morphological dependence exist for galaxy orientation?

This paper is organized as follows: in Sect. 2 we describe the sample used and the method of data reduction. In Sects. 3 and 4 we describe the methods and the selection effects. A discussion of the statistical results and conclusions are presented in Sects. 5 and 6.

\section{The sample}

We have compiled a database of 4637 galaxies in the LSC covering 20 catalogues. A catalogue needs to contain at least 10 LSC galaxies in order to be selected. A list of the catalogues are given in Table 1. The NASA/IPAC extragalactic database (NED, http: //nedwww . ipac . caltech. edu/) was used to compile these catalogues. The abbrevations given in the first column of Table 1 are as follows: NGC - New General Catalogue, UGC - Uppsala General Catalogue of Galaxies, ESO - ESO/Uppsala Survey of the ESO (B) Atlas, IC Index Catalogue, MCG - Morphological Galaxy Catalogue, UGCA - Uppsala obs. General Catalogue, Addendum, VCC - Virgo Cluster Catalogue, CGCG - Catalogue of Galaxies and Clusters of Galaxies, KUG - Kiso Ultraviolet Galaxy Catalogue, MRK - Markarian Galaxy Catalogue, MESSIER - Catalogue des nebuleuses et des amas d'etoiles, BCG

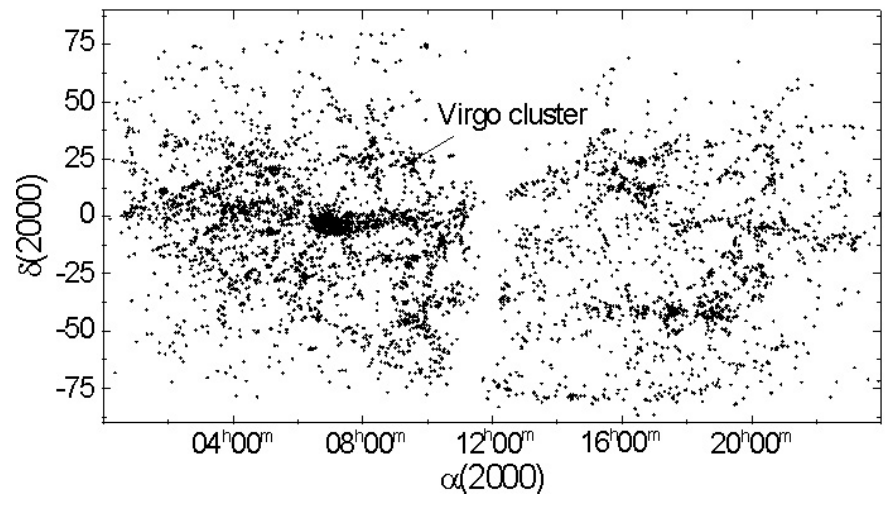

Fig. 1. All-sky distribution of 4637 LSC galaxies. The Virgo cluster can be seen.

- Brandner+Grebel+Chu Catalogue, LSBG - Low Surface Brightness Galaxies, SBS - Second Byurakan Survey, LCRS - Las Companas Red Shift Survey, DDO - David Dunlap Observatory Publications, IRAS - Infrared Astronomical Satellite, SGC - Southern Galaxy Catalogue, UM - University of Michigan: Curtis Schmidt-thin prism survey for extragalactic emission-line objects: List I-V and CCC - Catalogue of Coma Cluster Galaxies. The references for the catalogues are listed in the last column of Table 1.

A galaxy needs to fulfill the following two selection criteria in order to be selected: (1) radial velocity $(R V)<3000 \mathrm{~km} \mathrm{~s}^{-1}$ and; (2) diameter given. The main editing process was as follows: first, galaxies having $R V<3000 \mathrm{~km} \mathrm{~s}^{-1}$ were collected; the $R V \mathrm{~s}$ were taken from LCRS. The second step was to compile the maximum $(a)$ and minimum $(b)$ diameters. The diameters were taken from various catalogues. Then the position angles (PAs) of galaxies taken from the UGC, ESO and Third Reference Catalogue of Bright Galaxies (de Vaucouleurs et al. 1991, hereafter RC3) were added. The morphology and the magnitudes of galaxies were noted.

In the NED, 7371 galaxies having $R V \mathrm{~s}<3000 \mathrm{~km} \mathrm{~s}^{-1}$ were listed until the cutoff date (April 2003). The diameters were given for $6270(85.1 \%)$ galaxies. Both the diameters and PAs were listed for 3799 (51.5\%) galaxies. Morphological information was given in the catalogues for 4920 (66.7\%) galaxies.

To enlarge the database we measured the PAs of 1599 galaxies on red-sensitive ESO or POSSII prints by eye. A binocular microscope (25-fold magnification), a transparent glass with a thin line and a protector of $15 \mathrm{~cm}$ radius was used to measure the PA of the major diameter of a galaxy. The database compiled in this way comprises 4637 galaxies. Their all-sky distribution is shown in Fig. 1. The Virgo cluster can easily be seen in this figure. Our database includes 2280 spirals (S), 1162 barred spirals (SB), 631 type I irregulars (Irr), 202 ellipticals (E) and 362 lenticulars (S0). Figure 2a shows the morphological distributions of the LSC galaxies in our database.

For a study of the origin of angular momentum, the sample should be restricted to only those galaxies supported by rotation. S and SB galaxies e.g. are supported by rotation (Kashlinsky 1982; Fall 1982). Bright ellipticals are known to be supported by stellar random velocity dispersion, rather than by 

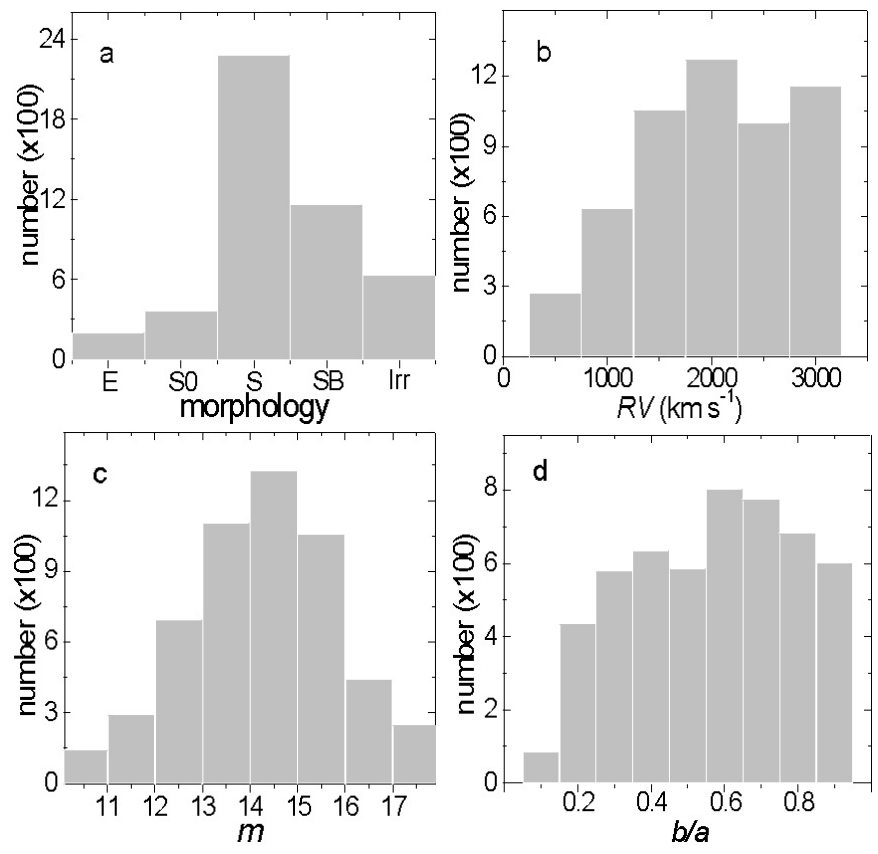

Fig. 2. The morphology a), radial velocity $R V$ b) axial ratios $b / a$ c) and the magnitude $m \mathbf{d}$ ) distributions of 4637 LSC galaxies.

rotation (Toomre \& Toomre 1972; Toomre 1977). Lenticulars (S0) exhibit some of the properties of both ellipticals and spirals and seem to bridge the two galaxy types (de Souza et al. 1985). Hence, we do not analyse the orientations of E and S0 galaxies, but devoted our attention to the subsamples of $\mathrm{S}$ and SB galaxies. Irregular type I galaxies, as a extension of spiral classes (beyond $\mathrm{Sd}$ ) were included in our analysis.

As subsamples of $\mathrm{S}$, and $\mathrm{SB}$ galaxies we used $\mathrm{Sa}, \mathrm{Sab}, \mathrm{Sb}$, $\mathrm{Sbc}, \mathrm{Sc}, \mathrm{Scd}, \mathrm{Sd}, \mathrm{Sdm}, \mathrm{Sm}$ and $\mathrm{SBa}, \mathrm{SBab}, \mathrm{SBb}, \mathrm{SBb}, \mathrm{SBc}$, SBcd, SBd, SBdm, SBm.

In our database, $83 \%$ of the galaxies have $R V \mathrm{~s}$ in the range 1000 to $3000 \mathrm{~km} \mathrm{~s}^{-1}$ and $85 \%$ of the galaxies have absolute magnitudes $\left(\mathrm{M}_{B}\right)$ in the range 12 to $16 \mathrm{mag}$. The histograms of the $R V$ and the magnitude $(m)$ distributions of the investigated 4637 LSC galaxies are shown in Figs. $2 b$ and c. Figure 2d shows the axial ratio $(b / a)$ distributions of the LSC galaxies. This histogram will be discussed in Sect. 4 .

\section{Method of analysis}

In the method of Hawley \& Peebles (1975), the observed PAs and axial ratio distributions are analysed independently. They also introduced the Fourier method of analysing PA histograms. A second approach was proposed by JS in which another important parameter, the inclination angle, was also taken into consideration. Later, FG corrected the inconsistencies in JS's approach. In the appendix of FG they describe how to derive the SV orientation of a galaxy and we followed their method. FG's method has been applied by several authors in galaxy orientation studies (KO; Go93; Go94; Hu et al. 1995; Wu et al. 1997; Yuan et al. 1997; Hu et al. 1998; Godlowski \& Ostrowski 1999; Aryal \& Saurer 2000 (AS); Aryal \& Saurer 2001; Flin 2001; Baier et al. 2003; Aryal \& Saurer 2004). In their method, the three dimensional orientation of the SV of a galaxy is characterized by two angles: the polar angle $(\theta)$ between the galactic SV and a reference plane, and the azimuthal angle $(\phi)$ between the projection of a galactic SV on to this reference plane and the $X$-axis within this plane. The reference plane is the LSC plane. The formulae to obtain $\theta$ and $\phi$ as given in $\mathrm{FG}$ are as follows:

$$
\begin{aligned}
& \sin \theta=-\cos i \sin B \pm \sin i \sin P \cos B \\
& \sin \phi=(\cos \theta)^{-1}[-\cos i \cos B \sin L+\sin i \\
& (\mp \sin P \sin B \sin L \mp \cos P \cos L)]
\end{aligned}
$$

where $L, B$ and $P$ are the supergalactic longitude, latitude and position angle, respectively. The angle $i$ is the inclination angle, estimated with Holmberg's (1946) formula: $\cos ^{2} i=\left[(b / a)^{2}-\right.$ $\left.0.2^{2}\right] /\left(1-0.2^{2}\right)$ with $b / a$ being the measured axial ratio. Here 0.2 is the assumed intrinsic flatness value for all types of disk galaxies. We adopt the supergalactic coordinate system as defined by Tammann \& Sandage (1976).

To calculate the angles $\theta$ and $\phi$ of a given disk galaxy, the measured quantities $\alpha, \delta$, PA (equatorial system) have to be transformed to the supergalactic variables $L, B, P$. The above formulae show that there are two possible solutions for a given galaxy. Considering the ambiguity of the rotation direction of the galactic disk, there are four solutions of the SV orientation for a galaxy. We count all four possibilities independently in our analysis.

We assume a spatial isotropic distribution of SVs of galaxies as a theoretical reference. This spatial reference distribution will give further reference distributions for the angles $\theta$ and $\phi$. As a next step, our observations are compared with these isotropic distribution curves in both $\theta$ and $\phi$. For this we use three different statistical tests: chi-square, Fourier, and auto correlation.

If the deviation from isotropy is only slowly varying with $\theta$ or $\phi$, the Fourier test can be applied. In this case the first order Fourier coefficient $\Delta_{11}$ determines the preferred orientation of SVs of galaxies with respect to the reference plane. For the $\theta$-distribution, a negative (positive) value of $\Delta_{11}$ indicates that the SVs of galaxies tend to lie parallel (perpendicular) to the LSC plane. In the $\phi$-distribution $\Delta_{11}>0$ means that the projections of SVs tend to point radially towards the LSC center. The first order Fourier probablity function $P\left(>\Delta_{1}\right)$ estimates whether a pronounced preferred orientation occurs in the sample (smaller value of $P\left(>\Delta_{1}\right)$ ) or not (higher value of $P\left(>\Delta_{1}\right)$ ).

For an isotropic distribution we expect the correlation function $C \rightarrow 0$. The critical limit is the standard deviation of the correlation coefficient $C$.

We set the chi-square probability function $P\left(>\chi^{2}\right)=0.050$ as the critical value to discriminate isotropy from anisotropy; this corresponds to a deviation from isotropy at the $2 \sigma$ level.

As conditions for anisotropy we set the following: chisquare probability $P\left(>\chi^{2}\right)<0.050$, correlation coefficient $C / \sigma(C)>1$, first order Fourier coefficient $\Delta_{11} / \sigma\left(\Delta_{11}\right)>1$ and the first order Fourier probability $P\left(>\Delta_{1}\right)>0.150$ as used by Go93. We assume weak anisotropy if $\Delta_{11} / \sigma\left(\Delta_{11}\right)<1.5$ and do not conclude anything for such samples or subsamples.

In all three statistical tests the bin size was chosen to be $10^{\circ}$ for equatorial PAs, $\theta$ and $\phi$-distributions. The ranges for the 
angles $\theta$ and $\phi$ are $0^{\circ}$ to $180^{\circ}$ (for $\theta+\pi / 2$ ) and $-90^{\circ}$ to $+90^{\circ}$ (for $\phi$ ), respectively. However, the range of the polar angle was reduced to $0^{\circ}-90^{\circ}$ by taking its absolute value (control test, FG 1986) and using this range in the histogram of the $\theta$-distribution. The statistically poor bins (number of solution $<5$ ) are omitted in the analysis.

The diameters given in the catalogues are measured by eye. These values lack objectivity, especially in the case of nearly face-on galaxies. In order to maintain homogeneity in the values of $a$ and $b$, at first, we selected sample galaxies from all 20 catalogues and then measured their diameters using a binocular microscope (25-fold magnification) on redsensitive POSSII prints. The conversion factors were estimated for each catalogue taking the diameters of sample galaxies in the Photometric Atlas of Northern Bright Galaxies (Kodaira et al. 1990, hereafter PANBG) as a reference. In PANBG, $b / a$ is given for 791 bright LSC galaxies which were measured by means of an ellipse-fitting technique applied to an isophote of $25 \mathrm{mag} \operatorname{arcsec}^{-2}$. In order to remove the Holmberg effect we adopt Fouque \& Paturel's (1985) method to convert measured diameters to standard photometric diameters.

The errors of the PAs are determined by comparing measured values with published ones and turned out to be less than $\pm 15^{\circ}$. Using the standard tool of error propagation, the errors in $i, \theta$, and $\phi$ can be estimated to be $5^{\circ}, 9^{\circ}$ and $12^{\circ}$, respectively.

\section{Selection effects}

The inhomogeneous distributions of positions $(L, B)$ of LSC galaxies can be seen in Figs. $3 a$ and $b$. The dashed curves in these figures represent the homogeneous distribution. The humps at $110^{\circ}$ in $L$ and at $-5^{\circ}$ in $B$-distributions, respectively are caused by Virgo cluster galaxies (Figs. 3a and b).

In the catalogues there were 1633 (22\%) face-on or nearly face-on galaxies. We could not add these LSC galaxies in our database because of the lack of data (diameters and PAs). In the axial ratio distributions, the decline at $0.70-0.90$ in Fig. $2 d$ is due to this selection effect against nearly face-on galaxies. This effect can also be seen very clearly in the inclination angle distribution. No galaxies can be seen in the first bin $\left(i<10^{\circ}\right)$ (Fig. 3c) and the number of galaxies in the first three bins is less than expected. The decline of the number of galaxies in the last two bins is probably due to the faint edge-on galaxies that could not be included in the database.

Hence, we noticed three kinds of selection effects in our database: (1) inhomogenous distribution of positions of galaxies; (2) lack of knowledge of PAs of nearly face-on galaxies; and (3) lack of edge-on galaxies. These are thus the main selection effects.

Aryal \& Saurer $(2000,2001)$ studied the results of random simulations for the expected isotropic distributions of the polar and azimuthal angles of galaxy rotation axes. They found that any selection criteria imposed on the data may cause severe changes in the shapes of the expected isotropic distribution curves. In order to remove the selection effects concerning positions and inclination angles we use the method described by AS. In their method, the spatial distribution of galaxy
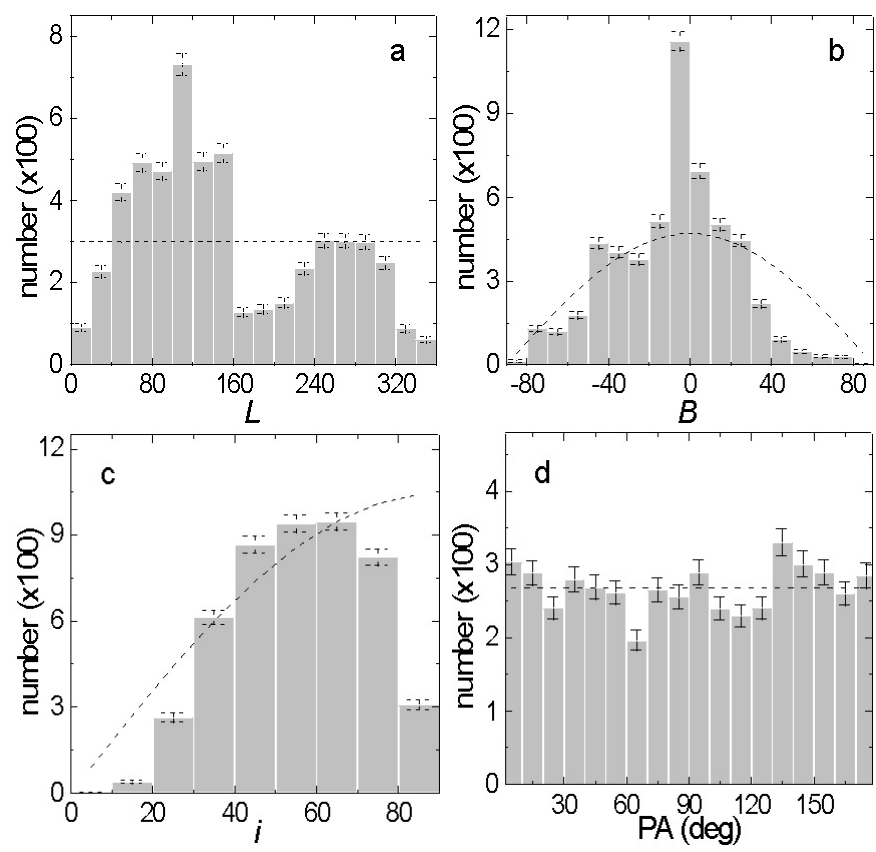

Fig. 3. The supergalactic longitude $L$ a), supergalactic latitude $B$ b), the inclination angle $i \mathbf{c}$ ), and equatorial PA distributions d) of 4637 LSC galaxies. The dashed curves represent the expected distributions. The observed values with statistical $\pm 1 \sigma$ error bars are shown.
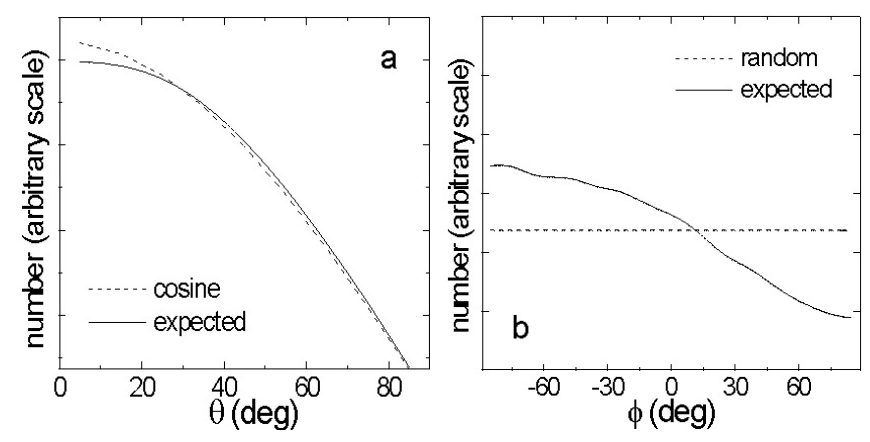

Fig. 4. The expected isotropic curves for polar $(\theta)$ and azimuthal $(\phi)$ angle of galaxy rotation axes. The solid lines represent the expected isotropic $\theta$ a) and $\phi$-distributions b) for 4637 LSC galaxies. The dashed lines represent the expected isotropic curves when there are no selections in the database.

rotation axes is assumed to be isotropic. Then, due to projection effects, $i$ is distributed $\propto \sin i, B$ can be distributed $\propto \cos B$, the variables $L$ and $P$ can be distributed randomly, and formulae (1) and (2) are used to calculate the corresponding values of $\theta$ and $\phi$.

To remove the selection effect concerning nearly face-on galaxies, the inclination angle was randomly distributed $\propto$ sine in the range $25^{\circ}$ to $85^{\circ}$ in the numerical simulation. The isotropic distribution curves are based on calculations including $1 \times 10^{6}$ virtual galaxies.

The expected isotropic distribution curves of $\theta$ and $\phi$ of our galaxies are shown in Fig. 4. In this figure, the dashed lines are the expected isotropic distribution curves when there is no selection on positions and PAs in the database. The expected $\theta$-distribution curve is found to hardly deviate from the cosine curve. According to AS, the isotropic polar angle distribution 
Table 2. Statistics of the polar angle distribution of LSC galaxies. The first two columns list the sample and the number of galaxies. The next columns give the chi-square probablity, correlation coefficient, first order Fourier coefficient, and first order Fourier probablity.

\begin{tabular}{llcccc}
\hline \hline Sample & $N$ & $P\left(>\chi^{2}\right)$ & $C / C(\sigma)$ & $\Delta_{11} / \sigma\left(\Delta_{11}\right)$ & $P\left(>\Delta_{1}\right)$ \\
\hline Total & 4073 & 0.027 & -1.1 & +1.1 & 0.189 \\
S & 2280 & 0.004 & -1.5 & +1.6 & 0.010 \\
SB & 1162 & 0.518 & -0.7 & +0.4 & 0.279 \\
Irr & 631 & 0.846 & +0.0 & +0.1 & 0.978 \\
\hline
\end{tabular}

is independent of $L$ but changes with $B$ when making selections on $i$. The deviation at small angles $\left(<15^{\circ}\right)$ in Fig. 4 a is due to the selections on inclination angles. The expected $\phi$-distribution curve remarkably deviates from the random distribution (Fig. 4b). This deviation is mainly due to the inhomogenous distributions of $L$. The azimuthal angle distribution changes with $L$ even when the range of $i$ and $B$ is full (AS).

The expected isotropic distribution curves (solid lines in Fig. 4) are compared with the observed distributions in the statistical analysis. We have used similar numerical methods to find the expected isotropic curves in the case of 8 Abell clusters in our latest paper (Aryal \& Saurer 2004)

\section{Results}

Figure $3 d$ shows the PA-distribution of our 4073 galaxies in the LSC together with statistical $\pm 1 \sigma$ error bars. In the figure, $P A=0^{\circ}$ or $180^{\circ}$ corresponds to the galactic rotation axes lying in the equatorial plane. The galaxies in the LSC show anisotropy in all three statistical tests. The chi-square probability $P\left(>\chi^{2}\right)$, the correlation coefficient $C / \sigma(C)$, first order Fourier coefficient $\Delta_{11} / \sigma\left(\Delta_{11}\right)$ and the first order Fourier probability $P\left(>\Delta_{1}\right)$ are found to be $0.000,2.5,+1.5$ and 0.131 , respectively. The positive $\Delta_{11}$ value at the $1.5 \sigma$ level suggests that the galactic planes of the galaxies in the LSC tend to be oriented perpendicular to the equatorial plane. The anisotropy is mainly due to the following deviations from the expected isotropic curves: humps at $5^{\circ}, 135^{\circ}$ and $155^{\circ}$ and dips at $65^{\circ}$, $105^{\circ}, 115^{\circ}$ and $125^{\circ}$.

This hint of anisotropy in the two-dimensional analysis prompted us to investigate morphological dependence in the galaxy orientation studies. The polar $(\theta)$ and azimuthal angle $(\phi)$ distributions (three-dimensional analysis) of LSC galaxies in the total sample and subsamples are given in Sects. 5.1 and 5.2. We compare our results to the recent published works in Sect. 5.3.

\subsection{Anisotropy in the polar angle distribution}

Table 2 lists the statistical parameters for the polar angle $(\theta)$ distribution of the total sample and the subsamples.

A common feature can be seen in the expected isotropic $\theta$ distribution curves (solid lines) in Fig. 5: a smooth dip at small angles with respect to the cosine curve (dashed line). This deviation is due to the selection effect against low inclination angle or nearly face-on galaxies. The shape of the expected isotropic
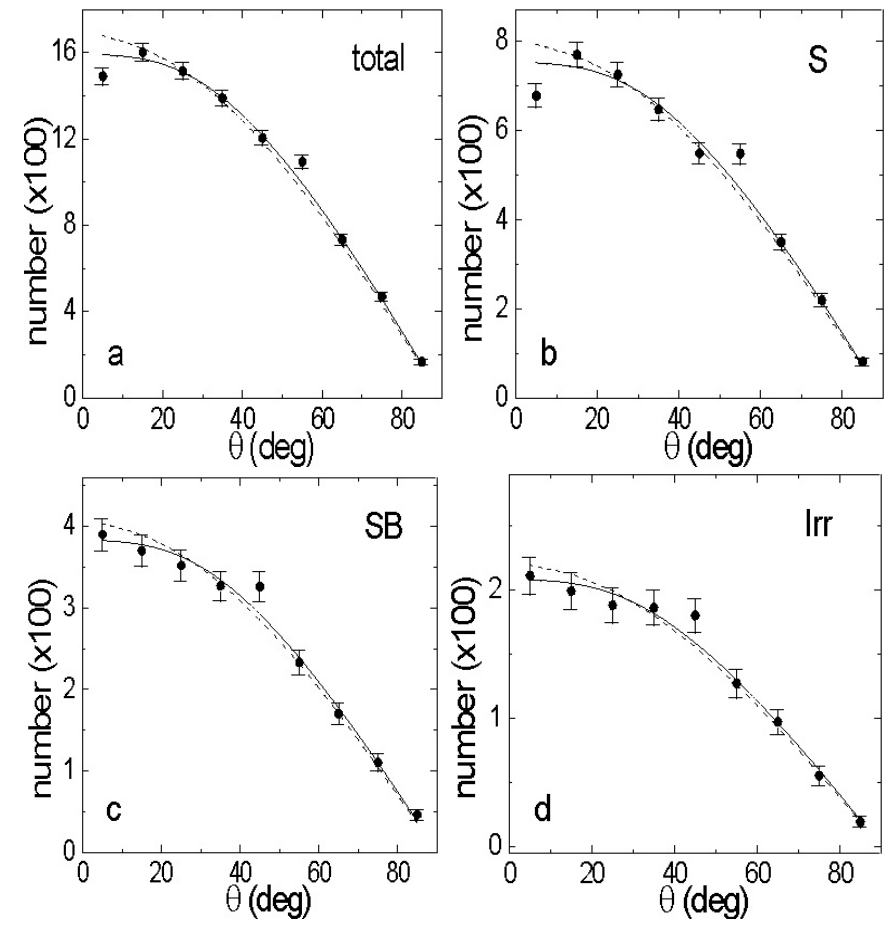

Fig. 5. The polar angle $(\theta)$ distribution of galaxies in the total sample a), S b), SB c) and Irr d). The solid line represents the expected isotropic distributions; the dashed lines give the cosine, for comparison. The observed counts with statistical $\pm 1 \sigma$ error bars are shown. $\theta=0^{\circ}$ corresponds to the galactic SV lying in the LSC plane.

$\theta$-distribution curves (solid lines in Fig. 5) are similar in the total sample and subsamples because of the similar selection effects concerning positions and inclination angles as explained by AS. In Fig. 5, $\theta=0^{\circ}$ corresponds to the case where galactic SVs tend to lie in the LSC plane.

In the total sample, the chi-square probability $P\left(>\chi^{2}\right)$ is found to be $2.7 \%$ and the correlation coefficient $C / \sigma(C)$ is -1.1 (Table 2). Both these statistics suggest a weak anisotropy. A small dip at $5^{\circ}(<1.5 \sigma)$ and a similar hump at $55^{\circ}(<1.5 \sigma)$ can be seen in Fig. 5a. This hump and dip cause the $\Delta_{11}$ value $<$ $1.5 \sigma$ error limit. Thus, we do not conclude anisotropy for the total sample.

The SB and the Irr galaxies show isotropy in all three statistics (Table 2). A hump at $45^{\circ}$ can be seen in both subsamples but it did not alter the statistical result as a whole (Figs. 5c and d). We will investigate the cause of this hump (at $45^{\circ}$ ) when analysing the subsamples of SB galaxies.

The only subsample which shows anisotropy is that of the spiral galaxies. All three statistical results show anisotropy (Table 2). A dip at $5^{\circ}$ and a hump at $55^{\circ}$ can be seen (Fig. 5b). This hump and dip cause the $\Delta_{11}$ value to be positive at a $1.6 \sigma$ level suggesting that the SVs of spiral galaxies in the LSC tend to be oriented perpendicular to the LSC plane. Thus, the galactic planes of the spiral galaxies in the LSC tend to lie parallel to the LSC plane.

It is noted that the humps and dips in the total sample and the subsample $\mathrm{S}$ are at similar angles: $5^{\circ}$ and $45^{\circ}$. The hump and the dip in the subsample $\mathrm{S}$ is deeper $(>2 \sigma)$ than in the total 
Table 3. Statistics of the polar angle distribution of the subsamples of spiral galaxies in the LSC. See Table 2 for an explanation of the columns.

\begin{tabular}{lrrrcc}
\hline \hline $\mathrm{T}$ & $N$ & $P\left(>\chi^{2}\right)$ & $C / C(\sigma)$ & $\Delta_{11} / \sigma\left(\Delta_{11}\right)$ & $P\left(>\Delta_{1}\right)$ \\
\hline $\mathrm{Sa}$ & 144 & 0.706 & +0.7 & -0.2 & 0.678 \\
$\mathrm{Sab}$ & 92 & 0.738 & -0.6 & +0.9 & 0.507 \\
$\mathrm{Sb}$ & 221 & 0.022 & +1.9 & +1.7 & 0.013 \\
$\mathrm{Sbc}$ & 197 & 0.725 & +0.4 & +1.0 & 0.273 \\
$\mathrm{Sc}$ & 292 & 0.003 & +1.3 & -0.2 & 0.878 \\
$\mathrm{Scd}$ & 217 & 0.000 & -1.5 & +1.8 & 0.086 \\
$\mathrm{Sd}$ & 156 & 0.515 & -0.9 & +0.4 & 0.732 \\
$\mathrm{Sdm}$ & 215 & 0.899 & -0.1 & +0.6 & 0.412 \\
$\mathrm{Sm}$ & 176 & 0.021 & +2.8 & -1.7 & 0.081 \\
\hline
\end{tabular}

sample. So, these two features in the total sample are due to the subsample $\mathrm{S}$.

Now we analyse the subsamples of $\mathrm{S}$ galaxies in more detail. The statistical results are given in Table 3. Figure 6 shows the $\theta$-distributions of the subsamples of $\mathrm{S}$ galaxies.

Three subsamples of S galaxies show anisotropy whereas 5 subsamples show isotropy in the statistics (Table 3). The subsample Sc shows an ambiguous result: anisotropy in the chisquare and auto-correlation tests and isotropy in the Fourier test. We discuss this subsample later.

A hump at $65^{\circ}$ is seen in the subsample Sb (Fig. 6c). Due to this hump $\Delta_{11}$ is found to be positive at the $1.7 \sigma$ level suggesting that the SVs of $\mathrm{Sb}$ galaxies tend to be oriented perpendicular to the LSC plane.

Similar to the subsample $\mathrm{Sb}$, the subsample Scd gives a positive $\Delta_{11}$ value at the $1.8 \sigma$ level (Table 3 ). The positive $\Delta_{11}$ is due to the dip at $5^{\circ}$ and $45^{\circ}$ and the humps at $55^{\circ}, 75^{\circ}$ and $85^{\circ}$ (Fig. 6f). Thus, Scd galaxies in the LSC show that their $\mathrm{SV}$ s tend to be oriented perpendicular to the LSC plane.

The subsample $\mathrm{Sm}$ shows an opposite orientation to that of $\mathrm{Sb}$ and Scd. In Fig. 6i, humps at $5^{\circ}, 15^{\circ}$ and dips at $55^{\circ}$ to $75^{\circ}$ can be seen. These humps and dips make the $\Delta_{11}$ value negative at the $1.8 \sigma$ level suggesting that the SVs of Sm galaxies in the LSC tend to lie parallel to the LSC plane.

Now we discuss the subsample Sc. In Fig. 6e, a hump at both small $\left(5^{\circ}\right)$ and large angles $\left(75^{\circ}\right.$ and $\left.85^{\circ}\right)$ can be seen. The hump at the small angle turns the $\Delta_{11}$ value negative whereas the hump at the large angle makes $\Delta_{11}$ positive. In the case of subsample Sc, $\Delta_{11}$ would be negative at a $2 \sigma$ level if there were no humps at $75^{\circ}$ and $85^{\circ}$. Similarly, $\Delta_{11}$ would be positive at a $2 \sigma$ level if there were no hump at $5^{\circ}$. A closer look at the mathematical expressions (Go93) clarifies that the humps and the dips at both small and large angles in the Fourier test compensate for each other in the mathematical analysis to show isotropy. Mathematically, $\Delta_{11}$ could be either positive or negative. We suspect that the Sc galaxies in the LSC show a bimodal tendency in the $\theta$-distribution.

The subsamples $\mathrm{Sa}$, Sab, Sbc, Sd and Sdm show isotropy in all three statistical tests. No deviation from an isotropic $\theta$ distribution curve is observed in these subsamples (Figs. 6a, b, $\mathrm{d}, \mathrm{g}$ and $\mathrm{h})$.
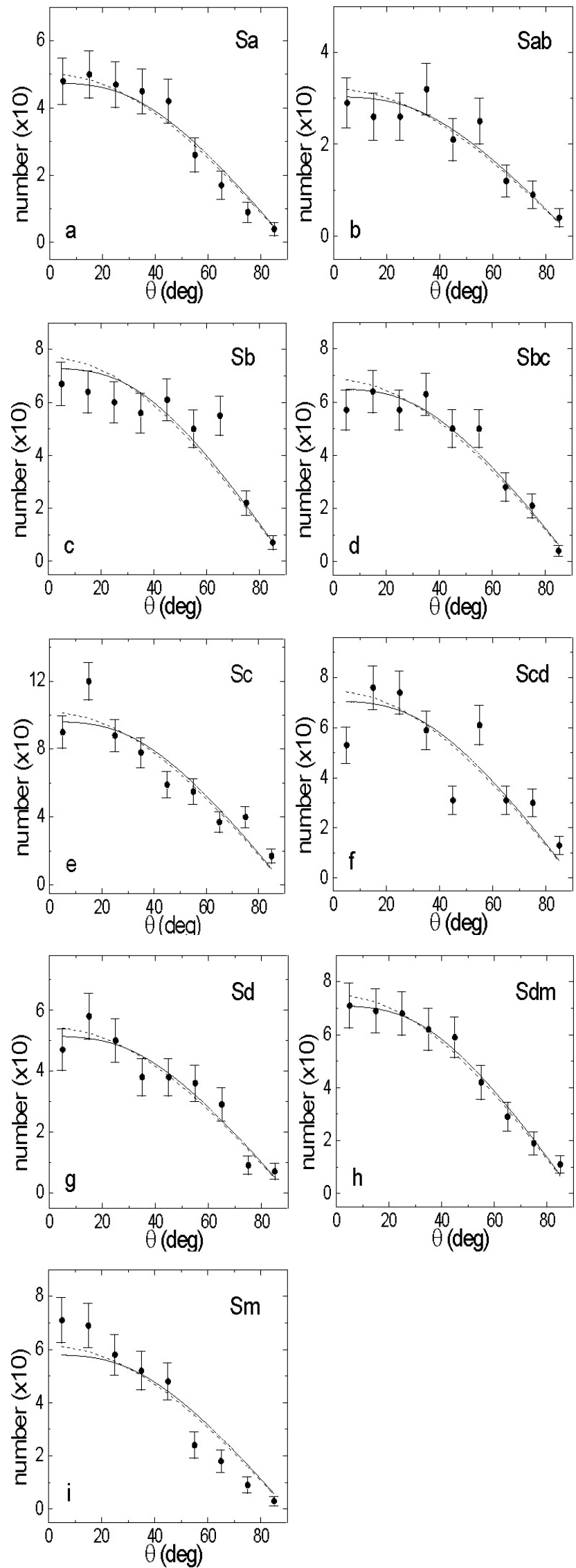

Fig. 6. The polar angle $(\theta)$ distribution of galaxies in $\mathrm{Sa} \mathbf{a})$, Sab b), $\mathrm{Sb} \mathbf{c}), \operatorname{Sbc} \mathbf{d}), \mathrm{Sc} \mathbf{e}), \mathrm{Scd} \mathbf{f}), \mathrm{Sd} \mathbf{g}), \mathrm{Sdm} \mathbf{h})$ and $\mathrm{Sm}$ i). This figure is analogous to Fig. 5 . 
Table 4. Statistics of the polar angle distribution of the subsamples of spiral barred galaxies in the LSC. See Table 2 for an explanation of the columns.

\begin{tabular}{lrrrcc}
\hline \hline$T$ & $N$ & $P\left(>\chi^{2}\right)$ & $C / C(\sigma)$ & $\Delta_{11} / \sigma\left(\Delta_{11}\right)$ & $P\left(>\Delta_{1}\right)$ \\
\hline SBa & 78 & 0.014 & -2.1 & -1.2 & 0.136 \\
SBab & 44 & 0.889 & -0.2 & -0.2 & 0.510 \\
SBb & 95 & 0.945 & +0.3 & +0.2 & 0.823 \\
SBbc & 72 & 0.954 & 0.0 & -0.3 & 0.784 \\
SBc & 129 & 0.018 & -1.8 & +1.1 & 0.104 \\
SBcd & 107 & 0.040 & +1.2 & +1.6 & 0.084 \\
SBd & 149 & 0.021 & -1.6 & +1.5 & 0.092 \\
SBdm & 124 & 0.992 & 0.0 & -0.3 & 0.948 \\
SBm & 182 & 0.882 & +0.1 & -0.5 & 0.734 \\
\hline
\end{tabular}

In the following we will discuss our results for the subsamples of SB. Table 4 gives the statistical results, Fig. 7 shows the $\theta$-distributions.

Two subsamples of SB galaxies show anisotropy in all three statistical tests (Table 4). These are the SBc and SBcd galaxies (Figs. 7e and f). Isotropy is found in seven subsamples of SB galaxies. In the subsample SBa a hump at $35^{\circ}$ can be seen (Fig. 7a). This hump makes the $\Delta_{11}$ value $<1.5 \sigma$ level which suggests a weak anisotropy. So, we do not conclude anisotropy for this subsample.

No preferred alignment is found for the subsample SBc. The subsamples SBcd and the SBd show anisotropy. In these subsamples dips at small angles $\left(<45^{\circ}\right)$ and humps at large angles $\left(>45^{\circ}\right)$ can be seen (Figs. $7 \mathrm{f}$ and $g$ ). Here $\Delta_{11}$ is found to be positive at the $1.5 \sigma$ level. This indicates that the SVs of SBcd and SBd galaxies in the LSC tend to be oriented perpendicular to the LSC plane.

The SBab, SBb, SBbc, SBdm and the SBm galaxies in the LSC show isotropy in all statistical tests (Table 4). No deviation from an isotropic $\theta$-distribution curve is noticed in the $\mathrm{SBab}$, $\mathrm{SBb}, \mathrm{SBbc}, \mathrm{SBdm}$ and the SBm LSC galaxies (Figs. 7b, c, d, h and i).

Thus, in the polar angle distributions we noticed an anisotropy for 6 samples and subsamples $-\Delta_{11} / \sigma\left(\Delta_{11}\right)$ is found to be positive in 5 cases - which indicates that the SV orientations of galaxies in the LSC tend to point perpendicular to the LSC plane.

\subsection{Anisotropy in the azimuthal angle distribution}

Statistical parameters for the azimuthal angle $(\phi)$ distribution of the LSC galaxies in the total sample and subsamples are given in Table 5.

The humps and dips in the histograms of the $\theta$-distribution are relatively easy to interpret. This is not the case with those of the $\phi$-distribution because of their ranges, $-90^{\circ}$ to $90^{\circ}$. In the histograms of the $\phi$-distribution, $\phi=0^{\circ}$ means the direction towards the Virgo cluster center (e.g. Fig. 8). Humps in the region $-50^{\circ}$ to $+50^{\circ}$ and the dips at $-90^{\circ}$ to $-50^{\circ}$ (first 4 bins) and at $50^{\circ}$ to $90^{\circ}$ (last 4 bins) make the $\Delta_{11}$ value positive in the $\phi$-distribution. A positive $\Delta_{11}$ suggests that the SV projections of galaxies tend to point radially towards the LSC center (or
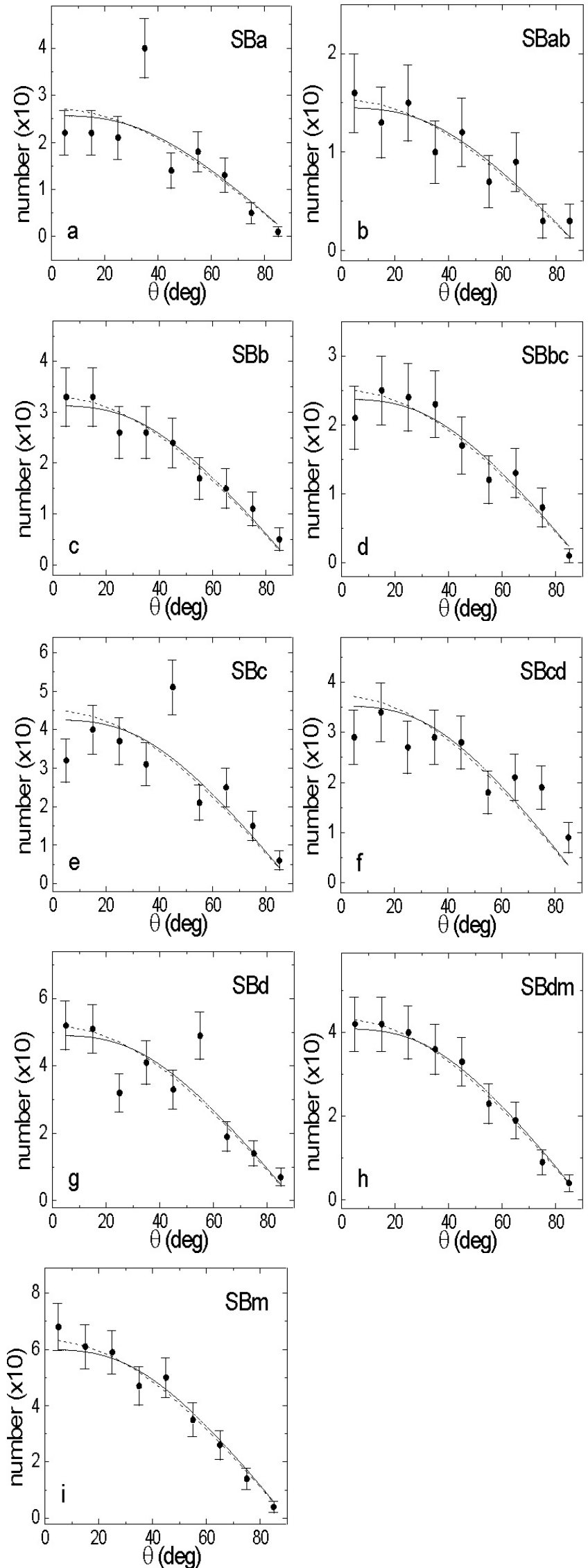

Fig. 7. The polar angle $(\theta)$ distribution of galaxies $\mathrm{SBa} \mathbf{a}), \mathrm{SBab} \mathbf{b}$ ), $\mathrm{SBb} \mathbf{c}), \mathrm{SBbc} \mathbf{d}), \mathrm{SBc}$ e), $\mathrm{SBcd} \mathbf{f}), \mathrm{SBd} \mathbf{g}), \mathrm{SBdm} \mathbf{h}$ ) and $\mathrm{SBm}$ i). This figure is analogous to Fig. 5. 
Table 5. Statistics of the azimuthal angle distribution of galaxies in the total sample and subsamples. See Table 2 for an explanation of the columns.

\begin{tabular}{lcccc}
\hline \hline Sample & $P\left(>\chi^{2}\right)$ & $C / C(\sigma)$ & $\Delta_{11} / \sigma\left(\Delta_{11}\right)$ & $P\left(>\Delta_{1}\right)$ \\
\hline total & 0.035 & +3.2 & -2.1 & 0.000 \\
S & 0.019 & +2.0 & -1.4 & 0.042 \\
SB & 0.990 & -0.4 & +0.5 & 0.738 \\
Irr & 0.034 & +2.8 & -1.6 & 0.040 \\
\hline
\end{tabular}
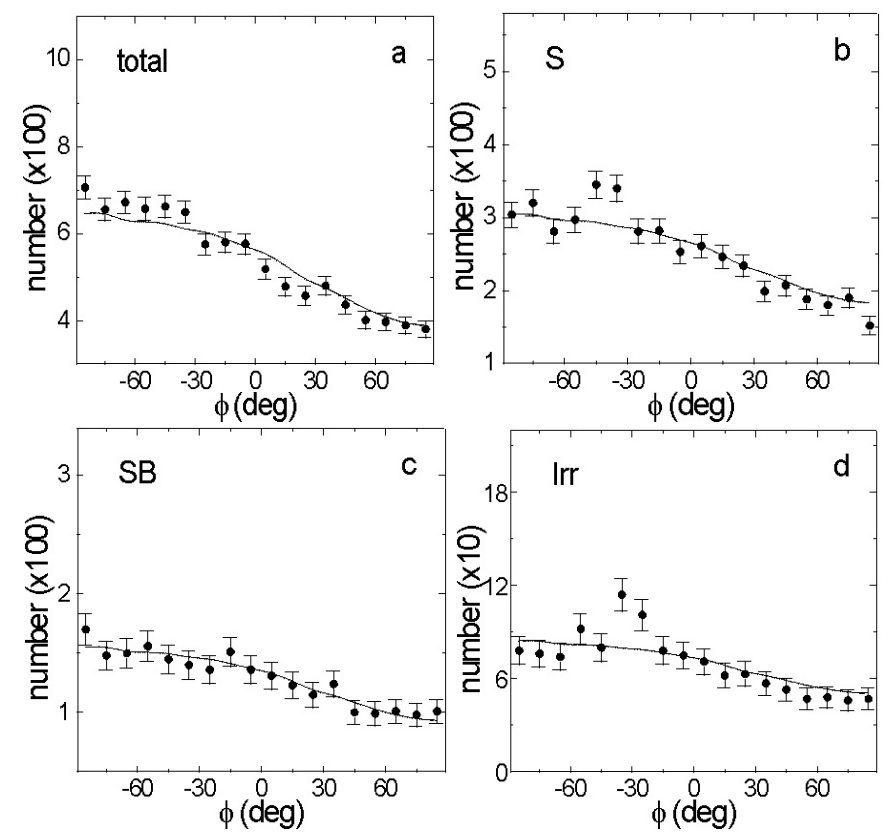

Fig. 8. The azimuthal angle $(\phi)$ distribution of galaxies in the total sample a), S b), SB c) and Irr d). The solid line represents the expected isotropic distributions; the dashed lines give the cosine, for comparison. The observed counts with statistical $\pm 1 \sigma$ error bars are shown. $\phi=0^{\circ}$ means the direction to the LSC center or the Virgo cluster center.

Virgo cluster center). Similarly, a negative $\Delta_{11}$ suggests that the SV projections of galaxies tend to orient tangentially with respect to the Virgo cluster center. In the $\phi$-distribution, the $\Delta_{11}$ value would be negative for $\phi$ when humps are at $-90^{\circ}$ to $-50^{\circ}$ (first 4 bins) and at $50^{\circ}$ to $90^{\circ}$ (last 4 bins) and dips are at $-50^{\circ}$ to $+50^{\circ}$.

Figure 8a shows the distribution of the SV projections $(\phi)$ of the 4073 galaxies in the LSC with respect to the Virgo cluster center. The total sample shows anisotropy in all three statistical tests (Table 5). A series of humps at $-85^{\circ},-65^{\circ},-55^{\circ}$ and dips at $5^{\circ}, 15^{\circ}, 25^{\circ}$ and $45^{\circ}$ make this sample have a negative $\Delta_{11}$ value at the $>2 \sigma$ level (see Fig. 8a and Table 5). This suggests that the SV projections of 4073 LSC galaxies tend to be oriented tangentially with respect to the Virgo cluster center.

Now we analyse the subsamples S, SB and Irr. The subsample SB shows isotropy in all three statistical tests. No humps or dips can be seen in Fig. 8c. Thus, the SV projections of SB galaxies in the LSC show a random orientation.

All three statistics show anisotropy for the $\mathrm{S}$ galaxies although not at a very high significance level. We do not conclude
Table 6. Statistics of the azimuthal angle distribution of the subsamples of spiral galaxies in the LSC. See Table 2 for an explanation of the columns.

\begin{tabular}{lcccc}
\hline \hline$T$ & $P\left(>\chi^{2}\right)$ & $C / C(\sigma)$ & $\Delta_{11} / \sigma\left(\Delta_{11}\right)$ & $P\left(>\Delta_{1}\right)$ \\
\hline $\mathrm{Sa}$ & 0.981 & +0.4 & +0.4 & 0.468 \\
$\mathrm{Sab}$ & 0.989 & -0.1 & +0.3 & 0.782 \\
$\mathrm{Sb}$ & 0.921 & +0.8 & -0.6 & 0.689 \\
$\mathrm{Sbc}$ & 0.020 & +2.4 & -1.3 & 0.120 \\
$\mathrm{Sc}$ & 0.999 & +0.2 & +0.3 & 0.687 \\
$\mathrm{Scd}$ & 0.013 & +1.3 & +1.8 & 0.076 \\
$\mathrm{Sd}$ & 0.968 & +1.0 & -0.5 & 0.521 \\
$\mathrm{Sdm}$ & 0.035 & +2.8 & +1.7 & 0.117 \\
$\mathrm{Sm}$ & 0.009 & +2.2 & -2.6 & 0.000 \\
\hline
\end{tabular}

anisotropy for this subsample because the $\Delta_{11}$ value is found to be $<1.5 \sigma$ level.

The humps at $-65^{\circ}$ and $-75^{\circ}$ cause the $\Delta_{11}$ value to be negative $(\sim 1.6 \sigma)$ in the subsample Irr (Fig. 8d). Hence the SV projections of irregular galaxies in the LSC tend to orient tangentially with respect to the Virgo cluster center. In the $\phi$ distribution, the irregular LSC galaxies show a similar alignment when compared to those of the total sample.

Three subsamples (Scd, Sdm and $\mathrm{Sm}$ ) of spiral galaxies show anisotropy and five subsamples show isotropy in the $\phi$ distribution. No clear indication is found for Sbc galaxies. The statistical results for the subsamples of spiral galaxies are given in Table 6. Figure 9 shows the distributions of SV projections of galaxies in the subsamples of LSC spirals.

For Scd and Sdm galaxies in the LSC a similar alignment is noticed. In these subsamples humps in the range $-50^{\circ}$ to $+50^{\circ}$ can be seen (Figs. 9f and h). Due to these humps the $\Delta_{11}$ values are found to be positive. This result suggests that the SV projections of Scd and Sm galaxies in the LSC tend to point radially towards the Virgo cluster center.

In the subsample Sbc a significant hump at $-55^{\circ}(>2 \sigma)$ can be seen. This hump makes the $\Delta_{11}$ value negative but not at a very high significance level (Fig. 9d). A significant hump at $-75^{\circ}$ causes Sm galaxies to show anisotropy in the $\phi$-distribution.

Now we analyse the $\phi$-distribution of the subsamples of SB galaxies. Two subsamples of SB galaxies show anisotropy and seven show isotropy in all three statistical tests (Table 7). The $\phi$-distributions in the subsamples of SB galaxies are shown in Fig. 10.

The SBa galaxies show anisotropy due to the humps at $-85^{\circ}$ and $-55^{\circ}$ (Fig. 10a). For this subsample the SV projections tend to point tangentially with respect to the Virgo cluster center.

The subsample $\mathrm{SBc}$ gives a positive $\Delta_{11}$ value in the Fourier test. The humps in the range $-50^{\circ}$ to $+50^{\circ}$ cause a positive $\Delta_{11}$ value (Figs. 10e). For SBc galaxies in the LSC the SV projections tend to point radially towards the Virgo cluster center.

The first order Fourier probability is found to be $60 \%$ and the $\Delta_{11}<1.5 \sigma$ for SBm. We do not conclude anisotropy for this subsample. 

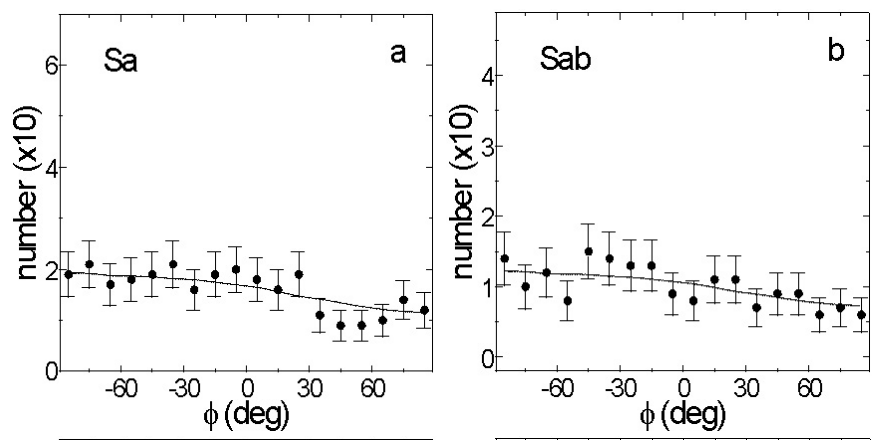

Table 7. Statistics of the azimuthal angle distribution of the subsamples of spiral barred galaxies in the LSC. See Table 2 for an explanation of the columns.

\begin{tabular}{lcrcc}
\hline \hline$T$ & $P\left(>\chi^{2}\right)$ & $C / C(\sigma)$ & $\Delta_{11} / \sigma\left(\Delta_{11}\right)$ & $P\left(>\Delta_{1}\right)$ \\
\hline SBa & 0.035 & +2.4 & -1.5 & 0.043 \\
SBab & 0.984 & 0.0 & -0.3 & 0.625 \\
SBb & 0.956 & +0.1 & +0.4 & 0.807 \\
SBbc & 0.997 & +0.1 & -0.6 & 0.582 \\
SBc & 0.036 & +4.3 & +1.8 & 0.016 \\
SBcd & 0.986 & +0.4 & +0.8 & 0.480 \\
SBd & 0.915 & +0.3 & +0.2 & 0.662 \\
SBdm & 0.994 & 0.0 & +0.1 & 0.982 \\
SBm & 0.014 & +1.9 & +1.4 & 0.602 \\
\hline
\end{tabular}

Table 8. A comparison with other recent work. The first 3 columns represent the references, samples and the results of other works. The fourth column gives our result. A comparison for the preferred alignment is given in the last column: "different" means $\theta(\phi)$ tends to lie parallel (radial) and perpendicular (tangential) with respect to the LSC plane (LSC center) and "identical" means our result is similar in all respects to other authors.
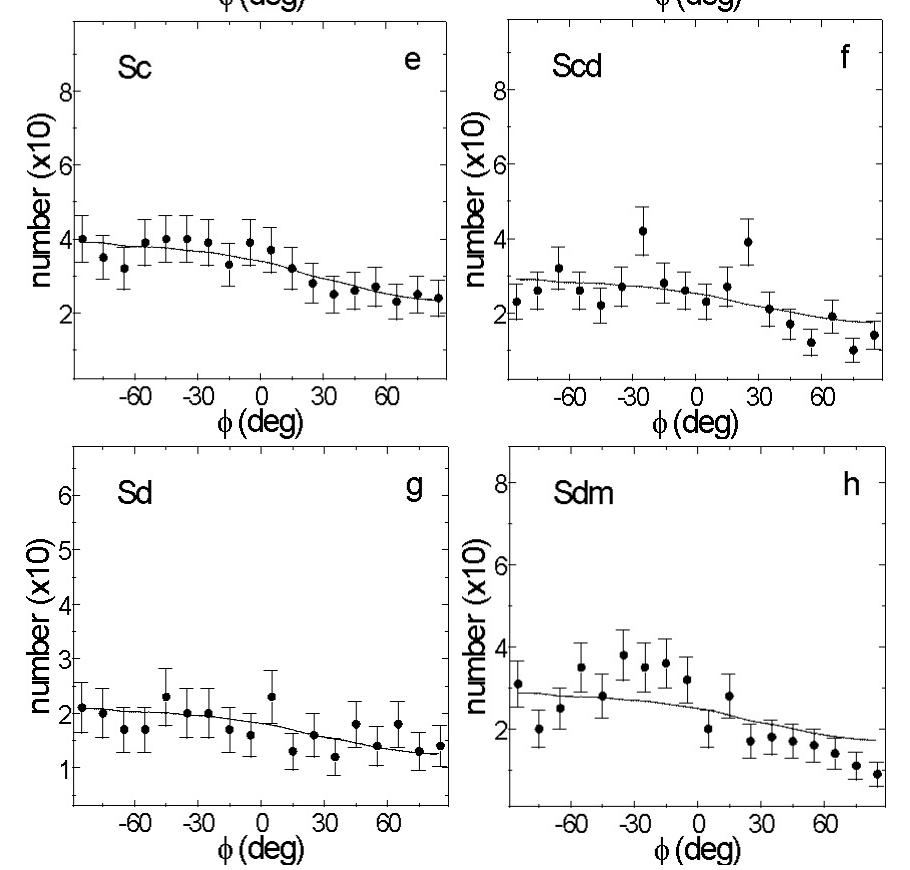

\begin{tabular}{|c|c|c|c|c|}
\hline Author & Sample & Result & Our result & Orientation \\
\hline \multirow[t]{8}{*}{ FG } & Sp I & $\theta:$ aniso & aniso & different \\
\hline & & $\phi$ : aniso & iso & - \\
\hline & Sp II & $\theta$ : iso & aniso & - \\
\hline & & $\phi:$ aniso & iso & - \\
\hline & Sp III & $\theta$ : iso & aniso & - \\
\hline & & $\phi$ : iso & iso & - \\
\hline & Sp IV & $\theta$ : iso & aniso & - \\
\hline & & $\phi$ : iso & iso & - \\
\hline \multirow[t]{4}{*}{$\mathrm{KO}$} & $6 a$ & $\theta$ : aniso & aniso & different \\
\hline & & $\phi:$ iso & iso & - \\
\hline & $6 \mathrm{~b}$ & $\theta:$ iso & aniso & - \\
\hline & & $\phi$ : iso & aniso & - \\
\hline \multirow[t]{4}{*}{ Go93 } & S & $\theta$ : iso & aniso & - \\
\hline & (UGC/ESO) & $\phi$ : iso & iso & - \\
\hline & $\mathrm{S}$ & $\theta$ : iso & aniso & - \\
\hline & (NGC-Tulley) & $\phi$ : iso & iso & - \\
\hline \multirow{2}{*}{$\begin{array}{l}\text { Hu et al. } \\
\text { (1995) }\end{array}$} & S & $\theta$ : aniso & aniso & identical \\
\hline & & $\phi$ : iso & iso & - \\
\hline
\end{tabular}

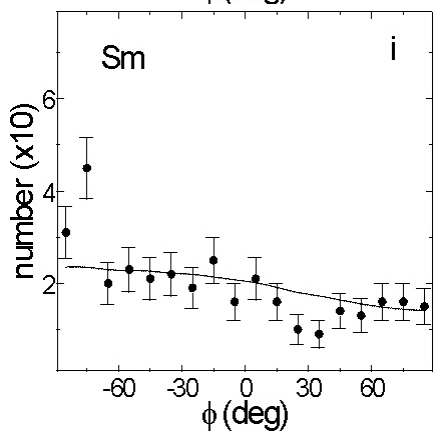

SBdm galaxies in the LSC show random orientations in the azimuthal angle distributions.

Thus, in the azimuthal angle distributions 7 samples and subsamples showed ansiotropy - the $\Delta_{11} / \sigma\left(\Delta_{11}\right)$ value is found to be positive in 3 cases and negative in 4 cases - i.e., no preferred alignment is noticed in the azimuthal angle distribution.

Fig. 9. The azimuthal angle ( $\phi$ ) distribution of galaxies $\mathrm{Sa}$ a), $\mathrm{Sab} \mathbf{b})$, $\mathrm{Sb} \mathbf{c}), \mathrm{Sbc} \mathbf{d}), \mathrm{Sc} \mathbf{e}), \mathrm{Scd} \mathbf{f}), \mathrm{Sd} \mathbf{g}), \mathrm{Sdm} \mathbf{h})$ and $\mathrm{Sm}$ i). This figure is analogous to Fig. 8.

No significant dips or humps can be seen in Figs. 10b, c, $\mathrm{d}, \mathrm{f}, \mathrm{g}$ and $\mathrm{h}$. Thus the SBab, SBb, SBbc, SBcd, SBd and the

\subsection{Comparison with other recent work}

In Table 8 we compare our results for the samples and subsamples with results for similar samples and subsamples of other works who have used a similar (PA-inclination) method to calculate polar and azimuthal angles of the galaxy rotation axes (FG; KO; Go93; Hu et al. 1995). 

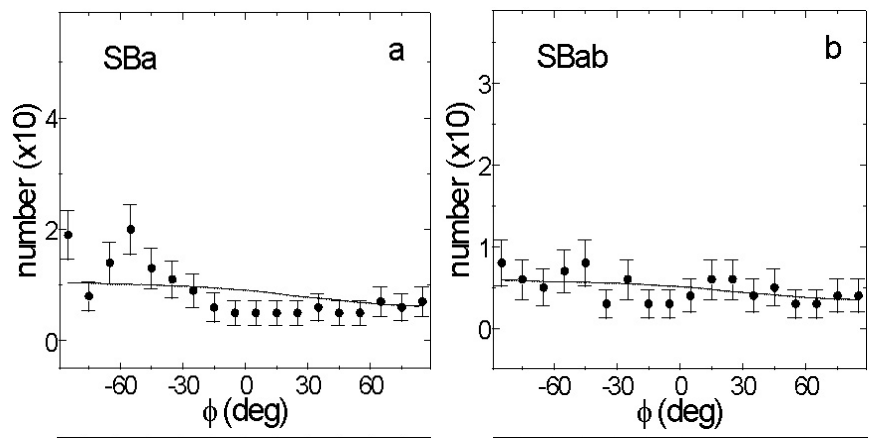

(all for $\phi$ ) and anisotropy in 3 (all for $\theta$ ). Our result does not agree with other recent work in 11 cases. We found a similar orientation in the $\theta$-distribution in only one case.

FG included all types of galaxies in their analysis and found a significant anisotropy. We refer to four "Sp" subsamples of FG which contain lenticulars and spirals (Table 5 of their paper). Among them, only the Sp I subsample (all spirals and lenticulars; $n=906$ ) shows anisotropy in their $\delta_{D}$-distribution, which is identical to the $\theta$-distribution in our notation. The anisotropy in Sp I is such that the SVs are parallel to the LSC plane. The remaining three subsamples (Sp II, Sp III and Sp IV) show isotropy (see Table 8). KO commented on FG's result for Sp I saying that the anisotropy is due to the large number of faint galaxies $(n=247)$ with uncertain or no PA in UGC. Our database for spirals is approximately 4 times and 2 times larger than that of $\mathrm{KO}$ and FG, respectively and selection effects due to face-on galaxies are removed in our analysis. We found anisotropy for spirals in $\theta$-distributions.

$\mathrm{KO}$ analysed the polar and azimuthal angle distributions of 618 galaxies in the LSC. KO noticed that the SV orientations of early-type spirals ( $T=0-4$, subsample $6 \mathrm{~b}$ ) tend to lie parallel to the LSC plane (see Fig. 5 in KO). We found, however, a different result: the SV orientations of early-type ( $\mathrm{Sb}$ ) galaxies tend to be oriented perpendicular to the LSC galaxies. The late-type spirals (subsample Sc and Scd) showed anisotropy in our analysis. KO obtained an opposite result for the latetype spirals ( $T=5-10$, subsample $6 \mathrm{c}$ ). In the $\phi$-distribution, KO found isotropy for both early- and late-type spirals. Our result is similar for the early-type spirals (subsample Sa, Sab and $\mathrm{Sb}$ ) in the $\phi$-distribution. In our work, four subsamples (Scd, Sdm and $\mathrm{Sm}$ ) of the late-type spirals show anisotropy in the $\phi$-distribution.

Now we consider the results of Go93. We compare our results for the subsample $\mathrm{S}$ to his subsample $\mathrm{S}$. Go93 analysed the $\mathrm{S}$ galaxies given in the UGC ESO Catalogue (Nilson 1973) and NGC Tully Catalogue (Tully 1988) separately. In the $\delta_{D}$-distribution ( $\theta$ in our notation) Go93 found isotropy for the spiral galaxies, but we obtained anisotropy. Go93 found anisotropy for the spirals (taken from the NGC Tully Catalogue) in the $\eta$-distribution ( $\phi$ in our notation) and we obtained a similar result for $\mathrm{S}$ galaxies although not at a very high significance level. The SV projections of spiral galaxies in the LSC tend to be oriented tangentially with respect to the Virgo cluster center. Go94 used an identical sample. Obviously, the results for $\mathrm{S}$ in Go93 are almost identical to those of Go94.

$\mathrm{Hu}$ et al. (1995) studied the orientations of 310 Virgo cluster galaxies. They found that the SV orientations of spiral galaxies tend to be oriented perpendicular to the LSC plane. We obtained a similar result when analysing 2280 LSC spirals.

\section{Conclusion}

10. The azimuthal angle $(\phi)$ distribution of galaxies $\mathrm{SBa}$ a), b), $\mathrm{SBb}$ c), $\mathrm{SBbc} \mathbf{d}), \mathrm{SBc}$ e), $\mathrm{SBcd} \mathbf{f}), \mathrm{SBd} \mathbf{g}), \mathrm{SBdm} \mathbf{h})$ and $\mathrm{SBm}$ i). This figure is analogous to Fig. 8.

In 7 cases ( 3 in $\theta, 4$ in $\phi$ ) our result is found to be similar to that of other authors (Table 8). Isotropy is found in 4 cases

Using the "PA-inclination" method we have analysed the spatial orientations of 4073 LSC galaxies in 22 samples and subsamples: the total sample, S total, SB total, irregulars, 9 subsamples of S, 9 subsamples of SB, each for polar and azimuthal 
angle, hence in total 44 cases. Isotropy is found in $31(70 \%$, out of 44) cases. In general our results support the hierarchical clustering scenario (Peebles 1969) which predicts that the directions of the spin vectors are entirely random.

In 13 cases we noticed anisotropy. We summarize this result as follows:

1. We found an anisotropic distribution of the SVs of spiral galaxies in the LSC and that the SV orientations of the spiral galaxies in the LSC tend to be oriented perpendicular to the LSC plane. The SV projections of these galaxies was found to be random. The SB galaxies showed isotropy in both polar and azimuthal angle distributions. Thus, the SVs orientations of SB galaxies are found to be randomly distributed.

2. The spatial orientations of early- $(\mathrm{Sb})$ and late-type spiral (Scd) galaxies are found to be identical. These galaxies show that their SVs tend to lie perpendicular to the LSC plane. In other words the galactic planes of $\mathrm{Sb}$ and $\mathrm{Scd}$ galaxies in the LSC tend to lie in the LSC plane whereas the galactic planes of Sm galaxies in the LSC tend to be oriented perpendicular to the LSC plane. The Sm galaxies in the LSC show an opposite alignment compared to that of $\mathrm{Sb}$ and $\mathrm{Scd}$ galaxies. The distributions of SV projections of early-type spirals is found to be random. The late-type spirals showed an ambiguous result in the azimuthal angle distribution.

3. The early-type SB galaxies show a random distribution whereas the late-type (SBcd, SBd) tend to be oriented perpendicular to the LSC plane. The SV projections of earlyand late-type galaxies show opposite alignment. The SV projections of late-type SB galaxies tend to point radially towards the center whereas the early-type tend to point tangentially with respect to the Virgo cluster center.

4. The LSC irregular galaxies show isotropy in the polar angle distributions and anisotropy in the azimuthal angle distributions, respectively. The SV projections of irregular galaxies in the LSC tend to orient tangentially with respect to the Virgo cluster center.

To understand the origin of these anisotropies requires further analysis. We intend to extend the analysis to richer clusters (e.g. Coma, Shapley Supercluster) and will discuss the origin of the angular momentum of galaxies in future work.

Thus, we suspect that the orientations of galaxies in the LSC could be closely related to galactic morphology.

Acknowledgements. The authors thank the referee A. Mazure whose remarks contributed to improve the paper. B. Aryal acknowledges the Oesterr. Akad. Austauschdienst (OeAD), Austria, for providing a scholarship and Tribhuvan University, Kathmandu, Nepal, for the study leave. We thank R. Weinberger, E. van Kampen and Y. Dixit for their helpful advice regarding the manuscript.

\section{References}

Aryal, B., \& Saurer, W. 2004, A\&A, 425, 871

Aryal, B., \& Saurer, W. 2001, in Galaxy Disk and Disk Galaxies, ed.

J. S. Funes \& E. M. Corsini, ASP Conf. Ser., 230, 145

Aryal, B., \& Saurer, W. 2000, A\&A, 364, L97 (AS)
Baier, F. W., Godlowski, W., \& MacGillivray, H. T. 2003, Ap\&SS, 403, 847

Bergh, V. D. 1959, Publ. David Dunlop Obs., 2, 145 (DDO)

Bergh, V. D. 1966, AJ, 71, 922 (DDO)

Binggeli, B., Sandage, A., \& Tammann, G. A. 1985, AJ, 90, 1681 (VCC)

Brandner, W., Grebel, E. K., Chu, Y.-H., et al. 2000, AJ, 119, 292 (BCG)

Corwin, H. G., de Vaucouleurs, A., \& de Vaucouleurs, G. 1985, Univ. Texas Monogr. Astron., 4, 1

Dekel, A. 1985, ApJ, 298, 461

de Souza, R. E., Vettolani, G., \& Chincarini, G. 1985, A\&A, 143, 143

de Vaucouleurs, G., de Vaucouleurs, A., Corwin, et al. 1991, Third Reference Catalogue of Bright Galaxies (New York: SpringerVerlag) (RC3)

Doi, M., Fukugita, M., Okamura, S., \& Tarusuwa, K. 1995, ApJS, 97, 77 (CCC)

Dreyer, J. L. E. 1895, MmRAS, 51, 185 (IC)

Dreyer, J. L. E. 1908, MmRAS, 59, 105 (IC)

Fall, S. M. 1982, in Progress in Cosmology; Proc. of the Oxford Intl. Symp. (Oxford, Dordrecht: D. Reidel Publishing Co.), 347

Flin, P., \& Godlowski W. 1986, MNRAS, 222, 525 (FG)

Flin, P. 2001, MNRAS, 325, 49

Fouque, P., \& Paturel, G. 1985, A\&A, 150, 192

Godlowski, W. 1993, MNRAS, 265, 874 (Go93)

Godlowski, W. 1994, MNRAS, 271, 19 (Go94)

Godlowski, W., Baier, F. W., \& MacGillivray, H. T. 1998, A\&A, 339, 709

Godlowski, W., \& Ostrosky, M. 1999, MNRAS, 303, 50

Hawley, D. L., \& Peebles, P. J. E. 1975, AJ, 80, 477

Helou, G. 1984, ApJ, 284, 471

Holmberg, E. 1946, Medd. Lund. Astron. Obs., Ser. VI, 117

Hu, F. X., Wu, G. X., Su, H. J., \& Liu, Y. Z. 1995, A\&A, 302, 45

Hu, F. X., Yuan, Q. R., Su, H. J., Wu, G. X., \& Liu, Y. Z. 1998, ApJ, 495,179

Impey, C. D., Sprayberry, D., Irwin, M. J., \& Bothun, G. D. 1996, ApJS, 105, 209

Jaaniste, J., \& Saar, E. 1978, in The Large Scale Structure of the Universe, ed. Longair M. S., Einasto J. (Dordrecht: Reidel), Proc. IAU Symp., 79, 448 (JS)

Kapranidis, N., \& Sullivan, W. T. 1983, A\&A, 118, 33

Kashikawa, N., \& Okamura, S. 1992, PASJ, 44, 493 (KO)

Kashlinsky, A. 1982, MNRAS, 200, 585

Kodaira, K., Okamura, S., \& Ichikawa, S. 1990, Photometric Atlas of Northern Bright Galaxies (Tokyo: Univ. of Tokyo Press) (PANBG)

Lauberts, A. 1982, ESO/Uppsala Survey of the ESO B Atlas (Garching bei Muenchen: ESO)

MacAlpine, G. M., Smith, S. B., \& Lewis, D. W. 1977a, ApJS, 34, 95 (UM)

MacAlpine, G. M., Smith, S. B., \& Lewis, D. W. 1977b, ApJS, 35, 197 (UM)

MacAlpine, G. M., Lewis, D. W., \& Smith, S. B. 1977c, ApJS, 35, 203 (UM)

MacAlpine, G. M., \& Lewis, D. W. 1978, ApJS, 36, 587 (UM)

MacAlpine, G. M., \& Williams, G. A. 1981, ApJS, 45, 113 (UM)

MacGillivray, H. T., \& Dodd, R. J. 1985, A\&A, 145, 269

MacGillivray, H. T., \& Dodd, R. J. 1985b, in ESO Workshop on Virgo Cluster 1984, ed. B. Binggeli (Garching bei Muenchen: ESO), 217

MacGillivray, H. T., McNally, B. V., Corwin, H. G., \& Dodd, R. J. 1982, MNRAS, 198, 605 
Markarian, B. E. 1967, Astrofizika, 3, 24 (MRK)

Markarian, B. E., Lipovetskii, V. A., \& Stepanian, D. A. 1983, Astrofizika, 19, 29

Messier, C. 1784, Catalogue des nébuleuses et des amas d'étoiles, Connaissance des Temps (MESSIER)

Nilson, P. 1973, Uppsala General Catalogue of Galaxies, Nova Acta Uppsala University, Ser. V:A, Vol. 1 (UGC)

Nilson, P. 1974, Upps. Astron. Obs. Rep., 5 (UGCA)

Peebles, P. J. E. 1969, ApJ, 155, 393

Reinhardt, M., \& Roberts, M. S. 1972, ApJ, 201, L12

Shectman, S. A., Landy, S. D., Oemler, A., et al. 1996, ApJ, 470, 172

Tammann, G. A., \& Sandage, A. 1976, ApJ, 207, L1

Takase, et al. 1980-2000, Kiso Ultraviolet Galaxy Catalog (Japan: Publ. Nat. Astron. Obs.)
Toomre, A., \& Toomre, J. 1972, ApJ, 178, 623

Toomre, A. 1977, in The Evolution of Galaxies and Stellar Populations, ed. B. Tinsley \& R. Larson, New Haven: Yale Univ. Obs.

Tully, R. B. 1988, Nearby Galaxy Catalog (Cambridge: Cambridge Univ. press)

Vorontsov-Vel, Y. B. A., Krasnogorskaya, A. A., \& Arkhipova, V. P. 1962-74, in Morphological Catalogue of Galaxies (Part I-V), Trudy Gosud. Astron. Inst. Shternberga (MGC)

Wu, G. X., Hu, F. X., Su, H. J., \& Liu, Y. Z. 1997, A\&A, 323, 317

Yuan, Q. R., Hu, F. X., Su, H. J., \& Huang, K. L. 1997, AJ, 114, 1308

Zwicky, F., Wild, P., Karpowicz, M., \& Kowal, C. T. 1961-68, The Catalogue of Galaxies and Clusters of Galaxies. California Inst. of Tech., Pasadena (CGCG) 